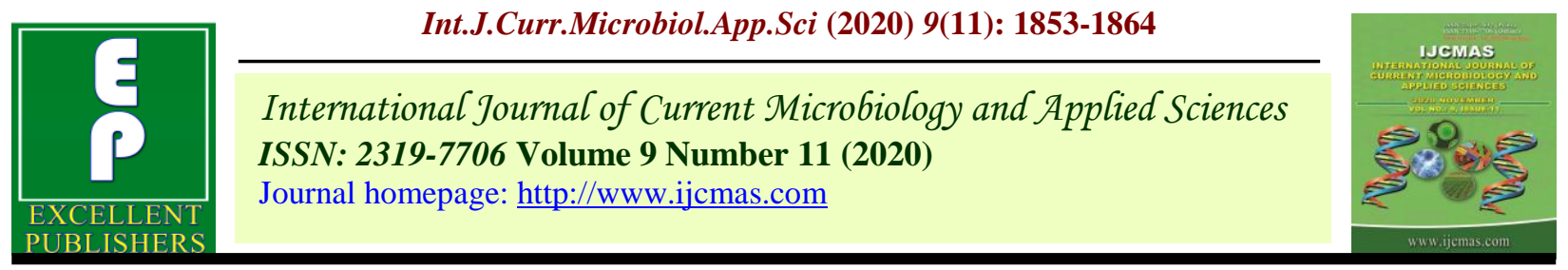

Original Research Article

https://doi.org/10.20546/ijcmas.2020.911.219

\title{
Effects of Egg Yolk and Egg Yolk Plasma Tris-Base Extenders on Beetal Buck Semen Preservation
}

\author{
Bipanpreet Singh $^{1 *}$, Daljeet Kaur ${ }^{1}$, Mandeep Singla ${ }^{1}$, Ranjna S. Cheema ${ }^{2}$, \\ A.P.S. Sethi ${ }^{3}$, Sumit Singhal ${ }^{4}$ and D.S. Malik ${ }^{1}$ \\ ${ }^{1}$ Department of Livestock Production Management, COVS, ${ }^{2}$ Department of Bioinfromatics, \\ ${ }^{3}$ Department of Animal Nutrition, ${ }^{4}$ Directorate Livestock Farms, GADVASU, \\ Ludhiana-141004, India \\ *Corresponding author
}

\section{A B S T R A C T}

Keywords

Egg yolk,

Plasma, Beetal buck semen preservation

Article Info

Accepted:

15 October 2020

Available Online:

10 November 2020
The present study was aimed to develop a most effective extender for long term storage of Beetal buck semen at $4^{\circ} \mathrm{C}$. Tris-citric acid-fructose was supplemented with egg yolk (TEY), egg yolk plasma (TEYP), @ 5, 10, 15 and 20; 5, 10, 15 and 20 percent, respectively. Extended semen was stored at $4^{\circ} \mathrm{C}$ and analyzed for sperm attributes. Values for motility, viability and plasma membrane integrity and acrosomal integrity remained significantly $(\mathrm{p}<0.05)$ higher in $15 \% \mathrm{TEY}, 15 \%$ TEYP from $0 \mathrm{hr}$ to $72 \mathrm{hrs}$ of storage. It indicated superiority of $15 \% \mathrm{TEY}, 15 \%$ TEYP, over other concentrations for storage of beetal buck semen at $4{ }^{\circ} \mathrm{C}$. A significant $(\mathrm{p}<0.05)$ decline in motility, viability and membrane integrity was observed during 0-72 hrs but sperm attributes were still $>50 \%$ at $72 \mathrm{hrs}$ of preservation. Though, both types of extenders at $15 \%$ level of concentration were able to sustain sperm attributes to $>60 \%$ level at $72 \mathrm{hrs}$ of preservation. Percent decline in sperm attributes was recorded comparatively less at $15 \%$ concentration in both type of extenders. Values for acrosome integrity also differed significantly $(p<0.05)$ among different concentrations of both types of extenders. In conclusion, both $15 \%$ TEY and 15\% TEYP were better than other concentrations.

\section{Introduction}

Goats are regarded as an intimate and integral part of rural farming system in India. About $27.8 \%$ of the total livestock is contributed by goats. The Goat population in the country in 2019 was 148.88 million showing an increase of $10.1 \%$ over the previous census $20^{\text {th }}$ Livestock Census (Anonymous, 2019). However, the recognized breeds of Indian goat population are facing a greater threat due to lack of enforcement of proper breeding policy, inadequate number of breeding bucks, indiscriminate crossbreeding and intermixing among breeds with local animals etc. Furthermore, the population of male bucks in the country in 2019 is 37.62 million is also showing a decrease of $14.65 \%$ over the previous census. To improve the productive potential of goat, the incorporation of superior 
germplasm into progeny is essential which is obviously possible with the use of outstanding sires. However, it is also estimated that there is a gap of $450 \%$ in availability of pure-bred pedigreed bucks (Singh et al., 2018). In rural areas, farmers usually have a small number of goats and also have limited access to a high quality sire or even a sire at all, due to which there is no breeding of the goat coming into heat through natural service. Due to all these reasons, continuous provision and maintenance of superior bucksis difficult and expensive either for natural mating or in existing network of AI centers all over the country.

\section{Materials and Methods}

Effect of different concentrations of egg yolk (EY), egg yolk plasma (EYP), supplemented to tris-citricacid-fructose buffer (T) was observed on beetal buck semen stored at $4^{\circ} \mathrm{C}$ from $0-72 \mathrm{hrs}$. EYP were purified from EY in the laboratory. Three ejaculates from each of the six bucks were analyzed for sperm attributes before initiating the actual trials. Eighteen trials per extender were performed at November, 2019 to March, 2020. Immediately after collection, volume, colour, consistency and mass activity of ejaculates were assessed. Volume was recorded in graduated tube and sperm concentration was calculated with help of haemocytometer. Semen was extended with Tris - citric acidfructose (TCF) buffer (Tris $3.08 \mathrm{~g}$; citric acid, $1.78 \mathrm{~g}$; fructose $1.25 \mathrm{~g} / 100 \mathrm{ml}$, gentamycin 5 mg, pH 7.2)having 5,10,15and $20 \%$ (v/v) EY and EYP. Samples having mass activity 4 and above were taken for study. Samples were divided into equal volumes and diluted with TCF (with different concentrations of EY and EYP). Sperm concentration was adjusted to $200 \times 10^{6} \mathrm{ml}^{-1}$ and diluted semen samples in tubes were shifted to a container containing warm water $\left(37^{\circ} \mathrm{C}\right)$ and placed in a cooling cabinet at $4^{\circ} \mathrm{C}$. Freshly extended and preserved semen at $4^{\circ} \mathrm{C}$ was analyzed for motility, viability, membrane and acrosome integrity after every $24 \mathrm{hrs}$ till $72 \mathrm{hrs}$ of storage.

Motility was evaluated by wet mount and track method. Viability was assessed by staining the spermatozoa using EosinNigrosin stain. Hypo-osmotic swelling test (HOST) was performed to analyze the integrity of sperm membrane. One drop (10 $\mu \mathrm{l})$ of semen incubated in $125 \mathrm{mOsm}$ hypoosmotic solution (HOS) at $37^{\circ} \mathrm{C}$ for $30 \mathrm{~min}$ was placed on a slide, covered with cover slip and examined under bright field microscope (olympus) at $400 \mathrm{X}$ for coiled tailed spermatozoa. The control was run in phosphate buffer saline (PBS, pH7.4). The number of coiled tailed spermatozoa in PBS was deducted from the number in HOS and the resultant figure was taken as the HOSreactive spermatozoa. For acrosomal integrity, sperm smear was prepared on clean glass slide and was stained using Giemsa stain. About 200 spermatozoa were counted in different fields for all the sperm attributes and percentage of motility, viability, plasma membrane integrity and acrosomal integrity was calculated.

\section{Statistical analysis}

The data recorded for experiment was analysized for statistical difference by analysis of variance (ANOVA) using SPSS20 programme and difference among treatments were compared as per Duncan's Multiple Range Test (Snedecor and Cochran, 1994)

\section{Results and Discussion}

\section{Effect of different concentration of egg yolk in Tris buffer on sperm attributes stored at $4^{\circ} \mathrm{C}$}

Table 1 depicts the values of attributes in the freshly extended semen and preserved semen 
at $4^{\circ} \mathrm{C}$ for $72 \mathrm{hrs}$ in Tris buffer supplemented with 5\%,10\%, 15\% and 20\% egg yolk (EY). Motility, viability, plasma membrane integrity (PMI) and acrosomal integrity (AcI) in freshly extended semen were significantly $(\mathrm{p}<0.05)$ higher in $15 \%$ compared to $5 \%$, $10 \%$ and $20 \%$ concentrations of triscitricacid-fructose- egg yolk (TCFEY) extender. A gradual decline $(p<0.05)$ was observed in motility, viability, PMI and AcI in the semen preserved at $4^{\circ} \mathrm{Cfor} 72 \mathrm{hrs}$ irrespective of egg yolk concentrations.

The values for sperm motility declined after $72 \mathrm{hrs}$ of preservation at different concentrations of egg yolk. The percent decline in motility was highest $(29.34 \%)$ at $5 \%$ conc. followed closely by $26.50 \%$ at $20 \%$ and $22.61 \%$ at $10 \%$ than least decline of $19.17 \%$ at $15 \%$ conc. of EY.

The percent decline in viability $(29.36 \%$, $26.38 \%, 21.09 \%$ and $19.36 \%)$, PMI (38.40\%, $32.64 \%, 31.26 \%$ and $25.54 \%$ ) and AcI $(28.15 \%, 25.55 \%, 22.22 \%$ and $18.90 \%)$ after $72 \mathrm{hrs}$ of preservation at different conc. of EY followed the same order. Overall, all the semen parameters showed less decline $(p<0.05)$ in $15 \%$ TFCEY extender compared to other concentrations indicated superiority of $15 \%$ TFCEY extender over the other tested extenders.

Al-Farque et al., (2007) compared four concentrations of egg yolk $(2.5,5.0,10 \&$ $15 \%)$ in citrate buffer and concluded that values for individual motility, AcI and normal morphology in frozen thaw semen were significantly higher in the presence of $10 \&$ $15 \%$ egg yolk.

Dias et al., (2014) also stated that $10 \%$ egg yolk in citrate extender provided higher viability to cryopreserved goat semen. It has been reported in previous studies that high concentration of egg yolk is detrimental to goat spermatozoa (Bispo et al., 2011).

In present study, $15 \%$ egg yolk concentration was better compared to $5,10 \& 20 \%$ to preserve buck semen at $4{ }^{\circ} \mathrm{C}$ for $72 \mathrm{hrs}$. The egg yolk at concentration of $10 \%$ was found suitable for freezing of goat semen in a study conducted by Parandekar et al., (1991). In Contrary, high post thaw motility was found in semen cryopreservation using $20 \%$ egg yolk in Tris based extender (Daskin and Tekin, 1996). Anand et al., (2017) also revealed that incorporation of $20 \%$ egg yolk had better cryopreservation for Barbari buck semen as reflected from motility, viability and AcI as compared to $3 \%$ egg yolk extender. Egg yolk is essential component of semen extender and acts as a non-penetrating cryoprotectant and prevents membrane damage. Its function may be defined to phospholipids/ cholesterol (Moce et al., 2010) and low density lipoprotein (Begeron and Manjunath, 2006). These components of egg yolk provide protection to sperm plasma membrane against cold shock. Besides protection to spermatozoa, egg yolk also reacts with seminal plasma glycoprotein-60 and phospholipase A2 to form toxic compound that lead to sperm death (Leboeuf et al., 2000). There is decline in sperm respiration with increased egg yolk concentration, which may lead to decline in sperm motility and viability (Bencharif et al., 2008, Najafi et al., 2013). Therefore, higher values of sperm attributes in 15\% TCFEY during the present study revealed that protective components of egg yolk were not sufficient in low concentration $(5,10 \%)$ to protect the sperm against cold shock. Since seminal plasma was removed in the present study, therefore phospholipase A2 and glycoprotein-60 may not be the reason for low sperm parameter in $20 \%$ egg yolk concentration during storage of semen at $4^{\circ} \mathrm{C}$ for 72 hrs. Rather, it seems that higher concentration of phospholipids, cholesterol and LDL in $20 \%$ egg yolk were responsible 
for significantly $(\mathrm{p}<0.05)$ declined parameters in 20\% TCFEY as compared to those in $15 \%$ TCFEY during present study. However, Siqueria et al., (2009) observed $62.50 \%$ motility in chilled semen using low concentration of egg yolk extender for $24 \mathrm{hrs}$.

The data for semen attributes using EYP at various concentrations for semen preservation at $4^{\circ} \mathrm{C}$ for $72 \mathrm{hrs}$ is given in Table 2 . The values of percent motility ranged between (80.50-83.67), viability (84.14-87.11), PMI (62.97-66.34) and AcI (90.80-92.63) and did not vary significantly ( $>0.05)$ among different concentration of TCFEYP (5-20\%) at $0 \mathrm{hr}$ (freshly extended semen) and at $24 \mathrm{hrs}$ of storage. However, percent motility, viability, PMI, AcI were significantly ( $\mathrm{p}<$ 0.05 ) higher at $15 \%$ conc. level. EYP as compared to other concentrations both at 48 $\mathrm{hrs}$ as well as at $72 \mathrm{hrs}$ of preservation.

The values for all the parameters were lowest in the presence of EYP at low conc. of 5\%. A gradual decline $(p<0.05)$ was also observed in motility, viability, PMI and AcI in the semen preserved at $4^{\circ} \mathrm{C}$ for $72 \mathrm{hrs}$ irrespective of egg yolk plasma concentrations with advancement in time of incubation.

Like the standardization of TCFEY, similar findings were obtained for motility, viability, PMI and AcI values which declined $(\mathrm{P}<0.05)$ less in 15\% TCFEYP $(11.34 \%, 11.36 \%$, $16.85 \%, 14.02 \%$ ) as compared to TCFEYP at 5,10 and $20 \%$ of concentrations.

There is no such research study regarding the effect of inclusion of EYP in buffer for preservation of goat semen. However, it has been demonstrated in Mongrel dog that inclusion of 20\% EYP in tris glucose buffer resulted in higher percentage of motility, viability, PMI, AcI to normal morphology compared to EY supplemented extender (Corcini et al., 2015).
Kaur et al., (2020) also revealed 15\% EYP in TCF buffer as the appropriate concentration for Labrador dos semen at $4^{\circ} \mathrm{C}$ for $72 \mathrm{hrs}$.

Effect of supplementation of egg yolk and egg yolk plasma in Tris-citric acid fructose extender for long term buck semen preservation at $4^{\circ} \mathrm{C}$

The egg yolk (EY) and egg yolk plasma (EYP) supplemented in Tris-citric acidfructose (TCF) extender at $15 \%$ concentration for semen preservation at $4^{\circ} \mathrm{C}$ for $72 \mathrm{hrs}$ were also compared for semen attributes (Table 3). The data indicated that all the semen parameters viz., motility, viability, plasma membrane integrity and Acrosomal integrity had significant difference, as the preservation time prolonged from $0 \mathrm{hrs}$ to 72 hrs irrespective the extender used. Both the types of extender had statistically similar results with respect to sperm attributes. Though EYP supplemented in Tris-citric acidfructose extender performed better especially for plasma membrane integrity with higher numerical value.

Kaur et al., (2020) reported that inclusion of $15 \%$ EY or EYP in TCF did not differ from each other with regard to sperm attributes, but visibility of spermatozoa was considerably better in EYP extender than EY extender which was attributed due to non-appearance of granules in EYP. Similar to the findings of Corcini et al., (2015), the values for motility (6.50\%), viability (5.37\%), PMI (6.97\%) and AcI (4.34\%) higher when compared with the values obtained for respective sperm attributes among both TCFEY and TCFEYP at $15 \%$ level of concentration. The higher values of EYP might be due to decreased in the level of seminal plasma protein, decreasing phospholipids and cholesterol during centrifugation of EYP which might be responsible for impairing cellular respiration and motility (Corcini et al., 2015). 
Table.1 Effect of egg yolk concentration in tris citric acid fructose buffer and preservation period on sperm attributes at $4{ }^{\circ} \mathrm{C}$

\begin{tabular}{|c|c|c|c|c|c|c|c|c|c|c|c|c|c|c|c|c|}
\hline \multirow{3}{*}{$\begin{array}{c}\text { Concen- } \\
\text { tration }\end{array}$} & \multicolumn{16}{|c|}{ Time period (hours) } \\
\hline & \multicolumn{4}{|c|}{0 hr } & \multicolumn{4}{|c|}{$24 \mathrm{hr}$} & \multicolumn{4}{|c|}{$48 \mathrm{hr}$} & \multicolumn{4}{|c|}{$72 \mathrm{hr}$} \\
\hline & Mot & Via & PMI & AcI & Mot & Via & PMI & AcI & Mot & Via & PMI & AcI & Mot & Via & PMI & AcI \\
\hline $5 \%$ & $\begin{array}{l}80.67^{b} \\
\pm 0.77\end{array}$ & $\begin{array}{l}84.24^{\mathrm{b}} \\
\pm 0.76\end{array}$ & $\begin{array}{c}62.95^{b} \\
\pm 1.05\end{array}$ & $\begin{array}{l}89.19^{c} \\
\pm 0.59\end{array}$ & $\begin{array}{l}68.66^{c} \\
\pm 0.78\end{array}$ & $\begin{array}{l}72.16^{c} \\
\pm 0.77\end{array}$ & $\begin{array}{l}47.11^{c} \\
\pm 0.98\end{array}$ & $\begin{array}{l}74.13^{d} \\
\pm 0.60\end{array}$ & $\begin{array}{l}59.50^{d} \\
\pm 0.75\end{array}$ & $\begin{array}{l}63.06^{c} \\
\pm 0.76\end{array}$ & $\begin{array}{l}37.02^{c} \\
\pm 1.67\end{array}$ & $\begin{array}{l}63.95^{\mathrm{c}} \\
\pm 0.60\end{array}$ & $\begin{array}{l}51.33^{\mathrm{d}} \\
\pm 0.74\end{array}$ & $\begin{array}{l}54.88^{c} \\
\pm 0.76\end{array}$ & $\begin{array}{l}24.55^{c} \\
\pm 0.98\end{array}$ & $\begin{array}{l}61.04^{d} \\
\pm 0.68\end{array}$ \\
\hline $10 \%$ & $\begin{array}{l}82.33^{b} \\
\pm 0.89\end{array}$ & $\begin{array}{l}84.47^{b} \\
\pm 1.67\end{array}$ & $\begin{array}{c}64.56^{\mathrm{ab}} \\
\pm 1.05\end{array}$ & $\begin{array}{c}91.37^{b} \\
\pm 0.57\end{array}$ & $\begin{array}{l}73.33^{\mathrm{b}} \\
\pm 1.01\end{array}$ & $\begin{array}{l}76.85^{\mathrm{b}} \\
\pm 1.00\end{array}$ & $\begin{array}{l}52.40^{\mathrm{b}} \\
\pm 1.13\end{array}$ & $\begin{array}{l}79.16^{\mathrm{b}} \\
\pm 0.69\end{array}$ & $\begin{array}{l}65.56^{\mathrm{b}} \\
\pm 1.24\end{array}$ & $\begin{array}{l}69.66^{\mathrm{b}} \\
\pm 1.00\end{array}$ & $\begin{array}{l}42.42^{\mathrm{b}} \\
\pm 1.18\end{array}$ & $\begin{array}{l}71.55^{b} \\
\pm 0.81\end{array}$ & $\begin{array}{l}59.72^{\mathrm{b}} \\
\pm 1.19\end{array}$ & $\begin{array}{c}63.38^{\mathrm{b}} \\
\pm 1.09\end{array}$ & $\begin{array}{l}33.30^{\mathrm{b}} \\
\pm 1.25\end{array}$ & $\begin{array}{l}69.15^{b} \\
\pm 0.83\end{array}$ \\
\hline $15 \%$ & $\begin{array}{l}85.00^{\mathrm{a}} \\
\pm 0.77\end{array}$ & $\begin{array}{l}88.47^{\mathrm{a}} \\
\pm 0.83\end{array}$ & $\begin{array}{l}67.80^{a} \\
\pm 1.60\end{array}$ & $\begin{array}{l}93.17^{\mathrm{a}} \\
\pm 0.49\end{array}$ & $\begin{array}{l}77.89^{a} \\
\pm 0.80\end{array}$ & $\begin{array}{l}81.40^{\mathrm{a}} \\
\pm 0.83\end{array}$ & $\begin{array}{l}59.47^{\mathrm{a}} \\
\pm 1.91\end{array}$ & $\begin{array}{l}82.86^{\mathrm{a}} \\
\pm 0.49\end{array}$ & $\begin{array}{l}71.78^{a} \\
\pm 0.84\end{array}$ & $\begin{array}{l}75.35^{\mathrm{a}} \\
\pm 0.83\end{array}$ & $\begin{array}{l}50.10^{\mathrm{a}} \\
\pm 1.09\end{array}$ & $\begin{array}{l}77.91^{a} \\
\pm 0.67\end{array}$ & $\begin{array}{l}65.83^{\mathrm{a}} \\
\pm 1.04\end{array}$ & $\begin{array}{l}69.38^{\mathrm{a}} \\
\pm 0.82\end{array}$ & $\begin{array}{l}42.54^{\mathrm{a}} \\
\pm 1.40\end{array}$ & $\begin{array}{l}74.27^{\mathrm{a}} \\
\pm 0.70\end{array}$ \\
\hline $20 \%$ & $\begin{array}{l}81.89^{b} \\
\pm 0.68\end{array}$ & $\begin{array}{l}85.33^{\mathrm{b}} \\
\pm 0.69\end{array}$ & $\begin{array}{c}64.75^{\mathrm{a} b} \\
\pm 1.16\end{array}$ & $\begin{array}{c}90.64^{b^{c}} \\
\pm 0.52\end{array}$ & $\begin{array}{l}70.72^{c} \\
\pm 0.71\end{array}$ & $\begin{array}{l}74.20^{c} \\
\pm 0.69\end{array}$ & $\begin{array}{l}52.50^{\mathrm{b}} \\
\pm 2.19\end{array}$ & $\begin{array}{l}76.56^{c} \\
\pm 0.52\end{array}$ & $\begin{array}{l}62.56^{c} \\
\pm 0.73\end{array}$ & $\begin{array}{l}66.08^{c} \\
\pm 0.69\end{array}$ & $\begin{array}{l}39.90^{\mathrm{c}} \\
\pm 1.09\end{array}$ & $\begin{array}{l}68.36^{c} \\
\pm 0.99\end{array}$ & $\begin{array}{l}55.39^{\mathrm{c}} \\
\pm 0.71\end{array}$ & $\begin{array}{l}58.95^{\mathrm{c}} \\
\pm 0.69\end{array}$ & $\begin{array}{l}32.11^{\mathrm{b}} \\
\pm 2.04\end{array}$ & $\begin{array}{l}65.09^{c} \\
\pm 0.57\end{array}$ \\
\hline Average & $\begin{array}{c}82.47^{\mathrm{A}} \\
\pm 0.43\end{array}$ & $\begin{array}{c}85.63^{\mathrm{A}} \\
\pm 0.56\end{array}$ & $\begin{array}{c}65.02^{\mathrm{A}} \\
\pm 0.58\end{array}$ & $\begin{array}{l}91.10^{\mathrm{A}} \\
\pm 0.32\end{array}$ & $\begin{array}{l}72.65^{\text {B }} \\
\pm 0.58\end{array}$ & $\begin{array}{c}76.15^{\mathrm{B}} \\
\pm 0.58\end{array}$ & $\begin{array}{c}52.87^{\mathrm{B}} \\
\pm 0.95\end{array}$ & $\begin{array}{l}78.18^{\mathrm{C}} \\
\pm 0.48\end{array}$ & $\begin{array}{l}64.85^{\mathrm{C}} \\
\pm 0.70\end{array}$ & $\begin{array}{c}68.53^{\mathrm{C}} \\
\pm 0.69\end{array}$ & $\begin{array}{l}42.36^{\mathrm{C}} \\
\pm 0.85\end{array}$ & $\begin{array}{l}70.45^{\mathrm{C}} \\
\pm 0.71\end{array}$ & $\begin{array}{c}58.07^{\mathrm{D}} \\
\pm 0.79\end{array}$ & $\begin{array}{l}61.65^{\mathrm{D}} \\
\pm 0.78\end{array}$ & $\begin{array}{c}33.13^{\mathrm{D}} \\
\pm 1.04\end{array}$ & $\begin{array}{c}67.39^{\mathrm{D}} \\
\pm 0.68\end{array}$ \\
\hline
\end{tabular}

Superscripts (A,B,C,D) indicate significant $(\mathrm{p}<0.05)$ effect of preservation time on sperm attributes. Superscripts $(\mathrm{a}, \mathrm{b}, \mathrm{c})$ indicate significant $(\mathrm{p}<0.05)$ effect of egg yolk concentration on sperm attributes.

Mot- Motility, Via- Viability, PMI- Plasma Membrane Integrity and AcI- Acrosome Integrity 
Table.2 Effect of Egg yolk plasma concentration in Tris citric acid fructose buffer and preservation period on sperm attributes at $4^{\circ} \mathrm{C}$

\begin{tabular}{|c|c|c|c|c|c|c|c|c|c|c|c|c|c|c|c|c|}
\hline \multirow{3}{*}{$\begin{array}{c}\text { Concen- } \\
\text { tration }\end{array}$} & \multicolumn{16}{|c|}{ Time period (hours) } \\
\hline & \multicolumn{4}{|c|}{$\mathbf{0}$} & \multicolumn{4}{|c|}{24} & \multicolumn{4}{|c|}{48} & \multicolumn{4}{|c|}{72} \\
\hline & Mot & Via & PMI & AcI & Mot & Via & PMI & AcI & Mot & Via & PMI & AcI & Mot & Via & PMI & AcI \\
\hline $5 \%$ & $\begin{array}{l}80.50^{\mathrm{a}} \\
\pm 1.43\end{array}$ & $\begin{array}{l}84.14^{\mathrm{a}} \\
\pm 1.40\end{array}$ & $\begin{array}{l}62.97^{\mathrm{a}} \\
\pm 1.21\end{array}$ & $\begin{array}{l}90.80^{\mathrm{a}} \\
\pm 1.27\end{array}$ & $\begin{array}{l}75.33^{\mathrm{a}} \\
\pm 3.32\end{array}$ & $\begin{array}{l}79.16^{\mathrm{a}} \\
\pm 3.27\end{array}$ & $\begin{array}{l}55.81^{\mathrm{a}} \\
\pm 1.48\end{array}$ & $\begin{array}{l}83.94^{\mathrm{a}} \\
\pm 3.95\end{array}$ & $\begin{array}{l}61.67^{c} \\
\pm 2.24\end{array}$ & $\begin{array}{l}68.12^{b} \\
\pm 2.33\end{array}$ & $\begin{array}{c}40.93^{c} \\
\pm 2.67\end{array}$ & $\begin{array}{l}70.84^{b} \\
\pm 2.57\end{array}$ & $\begin{array}{l}57.17^{\mathrm{c}} \\
\pm 1.44\end{array}$ & $\begin{array}{l}60.84^{\mathrm{c}} \\
\pm 1.39\end{array}$ & $\begin{array}{l}30.13^{c} \\
\pm 1.13\end{array}$ & $\begin{array}{l}65.04^{c} \\
\pm 1.19\end{array}$ \\
\hline $10 \%$ & $\begin{array}{l}81.67^{\mathrm{a}} \\
\pm 1.26\end{array}$ & $\begin{array}{l}85.25^{a} \\
\pm 1.36\end{array}$ & $\begin{array}{l}64.26^{\mathrm{a}} \\
\pm 1.09\end{array}$ & $\begin{array}{l}92.03^{\mathrm{a}} \\
\pm 1.12\end{array}$ & $\begin{array}{l}78.50^{\mathrm{a}} \\
\pm 2.71\end{array}$ & $\begin{array}{l}82.13^{a} \\
\pm 2.84\end{array}$ & $\begin{array}{c}59.24^{\mathrm{a}} \\
\pm 2.42\end{array}$ & $\begin{array}{c}85.85 \\
a \pm 3.90\end{array}$ & $\begin{array}{l}70.00 \\
\mathrm{ab} \\
\pm 1.46\end{array}$ & $\begin{array}{c}\underset{a b}{73.59} \\
\pm 1.55\end{array}$ & $\begin{array}{c}46.82^{b} \\
\pm 0.85\end{array}$ & $\begin{array}{c}76.29 \\
a b \\
\pm 1.22\end{array}$ & $\begin{array}{c}64.83^{b} \\
\pm 2.09\end{array}$ & $\begin{array}{l}68.29^{b} \\
\pm 2.12\end{array}$ & $\begin{array}{c}40.01^{b} \\
\pm 0.74\end{array}$ & $\begin{array}{l}72.07^{\mathrm{b}} \\
\pm 1.04\end{array}$ \\
\hline $15 \%$ & $\begin{array}{l}83.67^{\mathrm{a}} \\
\pm 1.26\end{array}$ & $\begin{array}{l}87.11^{a} \\
\pm 1.36\end{array}$ & $\begin{array}{l}66.34^{a} \\
\pm 0.80\end{array}$ & $\begin{array}{l}92.63^{\mathrm{a}} \\
\pm 1.14\end{array}$ & $\begin{array}{l}81.50^{\mathrm{a}} \\
\pm 2.10\end{array}$ & $\begin{array}{l}85.02^{a} \\
\pm 2.18\end{array}$ & $\begin{array}{c}62.35^{\mathrm{a}} \\
\pm 1.48\end{array}$ & $\begin{array}{l}89.19^{a} \\
\pm 2.47\end{array}$ & $\begin{array}{l}75.00^{\mathrm{a}} \\
\pm 1.36\end{array}$ & $\begin{array}{l}78.37^{\mathrm{a}} \\
\pm 1.42\end{array}$ & $\begin{array}{l}52.26^{\mathrm{a}} \\
\pm 0.81\end{array}$ & $\begin{array}{l}81.13^{\mathrm{a}} \\
\pm 1.27\end{array}$ & $\begin{array}{l}72.33^{a} \\
\pm 1.15\end{array}$ & $\begin{array}{l}75.75^{a} \\
\pm 1.19\end{array}$ & $\begin{array}{l}49.49^{a} \\
\pm 1.62\end{array}$ & $\begin{array}{l}78.61^{\mathrm{a}} \\
\pm 0.84\end{array}$ \\
\hline $20 \%$ & $\begin{array}{l}81.67^{\mathrm{a}} \\
\pm 1.43\end{array}$ & $\begin{array}{c}85.07^{\mathrm{a}} \\
\pm 1.45\end{array}$ & $\begin{array}{l}63.77^{a} \\
\pm 1.34\end{array}$ & $\begin{array}{l}91.50^{\mathrm{a}} \\
\pm 1.25\end{array}$ & $\begin{array}{l}77.00^{\mathrm{a}} \\
\pm 3.07\end{array}$ & $\begin{array}{l}80.60^{\mathrm{a}} \\
\pm 3.03\end{array}$ & $\begin{array}{l}57.81^{\mathrm{a}} \\
\pm 3.12\end{array}$ & $\begin{array}{l}85.99^{a} \\
\pm 3.42\end{array}$ & $\begin{array}{l}65.17^{\mathrm{b}} \\
\pm 2.02\end{array}$ & $\begin{array}{l}68.63^{b} \\
\pm 2.04\end{array}$ & $\begin{array}{c}43.41^{\mathrm{c}} \\
\pm 2.28\end{array}$ & $\begin{array}{l}74.52^{b} \\
\pm 2.38\end{array}$ & $\begin{array}{l}61.00^{c} \\
\pm 1.65\end{array}$ & $\begin{array}{l}64.13^{c} \\
\pm 1.68\end{array}$ & $\begin{array}{l}33.78^{c} \\
\pm 1.42\end{array}$ & $\begin{array}{l}67.90^{\mathrm{c}} \\
\pm 1.68\end{array}$ \\
\hline Average & $\begin{array}{c}81.88^{\mathrm{A}} \\
\pm 0.67\end{array}$ & $\begin{array}{c}85.40^{\mathrm{A}} \\
\pm 0.69\end{array}$ & $\begin{array}{c}64.33^{\mathrm{A}} \\
\pm 0.59\end{array}$ & $\begin{array}{c}91.74^{\mathrm{A}} \\
\pm 0.58\end{array}$ & $\begin{array}{c}78.08^{\mathrm{B}} \\
\pm 1.40\end{array}$ & $\begin{array}{l}81.73^{\text {В }} \\
\pm 1.41\end{array}$ & $\begin{array}{c}58.80^{\mathrm{B}} \\
\pm 1.37\end{array}$ & $\begin{array}{c}86.24^{\mathrm{B}} \\
\pm 1.67\end{array}$ & $\begin{array}{c}67.95^{\mathrm{C}} \\
\pm 1.35\end{array}$ & $\begin{array}{c}72.18^{\mathrm{C}} \\
\pm 1.23\end{array}$ & $\begin{array}{c}45.86^{\mathrm{C}} \\
\pm 1.23\end{array}$ & $\begin{array}{c}75.70^{C} \\
\pm 1.20\end{array}$ & $\begin{array}{c}63.83^{\mathrm{D}} \\
\pm 1.39\end{array}$ & $\begin{array}{l}67.25^{\mathrm{D}} \\
\pm 1.39\end{array}$ & $\begin{array}{c}38.35^{\mathrm{D}} \\
\pm 1.64\end{array}$ & $\begin{array}{l}70.91^{\mathrm{D}} \\
\pm 1.21\end{array}$ \\
\hline
\end{tabular}

Superscripts $(A, B, C, D)$ indicate significant $(p<0.05)$ effect of preservation time on sperm attributes. Superscripts $(a, b, c)$ indicate significant $(\mathrm{p}<0.05)$ effect of egg yolk concentration on sperm attributes.

Mot- Motility, Via- Viability, PMI- Plasma Membrane Integrity and AcI- Acrosome Integrity 
Table.3 Effect of supplementation of egg yolk and egg yolk plasma to Tris-ciric acid fructose extender for long term buck semen preservation at $4^{\circ} \mathrm{C}$

\begin{tabular}{|c|c|c|c|c|c|c|c|c|}
\hline \multirow[b]{2}{*}{$\begin{array}{l}\text { Sperm } \\
\text { attributes }\end{array}$} & \multicolumn{2}{|c|}{ O hr } & \multicolumn{2}{|c|}{$24 \mathrm{hr}$} & \multicolumn{2}{|c|}{$48 \mathrm{hr}$} & \multicolumn{2}{|c|}{$72 \mathrm{hr}$} \\
\hline & $\mathrm{EY}$ & EYP & $\mathrm{EY}$ & EYP & $\mathrm{EY}$ & EYP & $\mathrm{EY}$ & EYP \\
\hline Motility\% & $\begin{array}{l}85.33^{\mathrm{a}} \\
\pm 0.66\end{array}$ & $\begin{array}{c}85.56^{\mathrm{a}} \\
\pm 0.56\end{array}$ & $\begin{array}{l}79.17^{b} \\
\pm 0.40\end{array}$ & $\begin{array}{c}80.50^{b} \\
\pm 0.43\end{array}$ & $\begin{array}{l}73.67^{\mathrm{c}} \\
\pm 0.42\end{array}$ & $\begin{array}{l}75.50^{c} \\
\pm 0.43\end{array}$ & $\begin{array}{l}69.67^{d} \\
\pm 0.31\end{array}$ & $\begin{array}{l}71.50^{d} \\
\pm 0.43\end{array}$ \\
\hline Viability \% & $88.63^{\mathrm{a}} \pm 0.38$ & $\begin{array}{c}89.09^{\mathrm{a}} \\
\pm 0.44\end{array}$ & $\begin{array}{c}82.65^{b} \pm \\
0.39\end{array}$ & $\begin{array}{l}84.01^{b} \\
\pm 0.45\end{array}$ & $\begin{array}{l}77.15^{\mathrm{c}} \\
\pm 0.31\end{array}$ & $\begin{array}{l}79.23^{c} \\
\pm 0.42\end{array}$ & $\begin{array}{l}72.77^{d} \\
\pm 0.37\end{array}$ & $\begin{array}{l}75.21^{d} \\
\pm 0.32\end{array}$ \\
\hline AcI \% & $90.79^{a} \pm 0.57$ & $\begin{array}{c}91.49^{\mathrm{a}} \\
\pm 0.48\end{array}$ & $\begin{array}{l}82.01^{b} \\
\pm 0.50\end{array}$ & $\begin{array}{c}83.29^{b} \\
\pm 0.47\end{array}$ & $\begin{array}{l}76.77^{\mathrm{c}} \\
\pm 0.50\end{array}$ & $\begin{array}{l}78.36^{\mathrm{c}} \\
\pm 0.48\end{array}$ & $\begin{array}{l}73.24^{d} \\
\pm 0.48\end{array}$ & $\begin{array}{l}75.16^{\mathrm{d}} \\
\pm 0.48\end{array}$ \\
\hline
\end{tabular}

Different superscripts in a row indicate significant $(\mathrm{p}<0.05)$ difference.

PMI- Plasma Membrane Integrity and AcI- Acrosome Integrity 
Fig.1 Effect of different concentration of egg yolk on sperm motility (A), viability (B), plasma membrane integrity $(\mathrm{C})$, Acrosomal integrity (D) stored at $4^{\circ} \mathrm{Cat}$ different interval of time
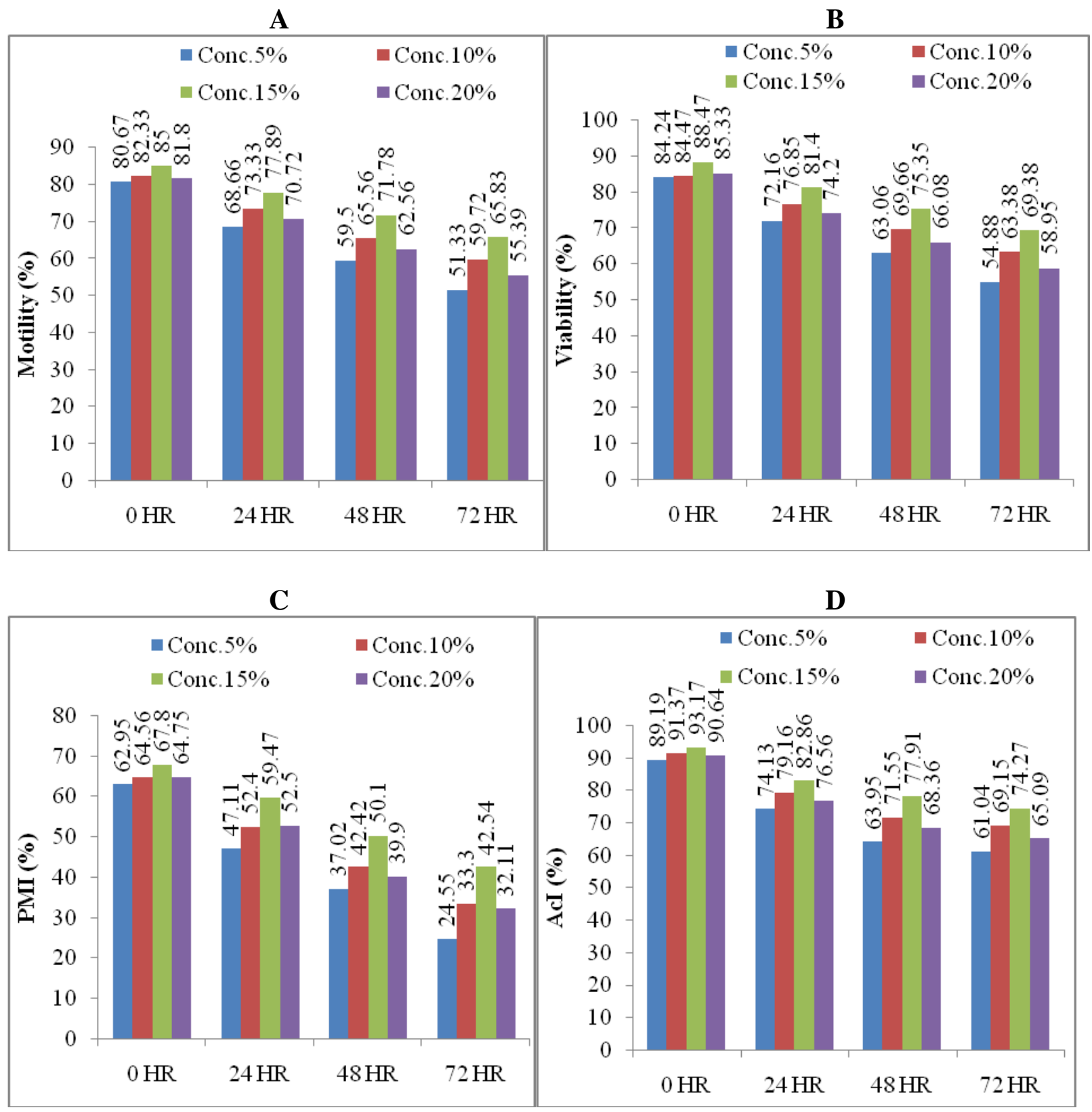
Fig.2 Effect of supplementation of egg yolk and egg yolk plasma in Tris-citric acid fructose extender for long term buck semen preservation at $4^{\circ} \mathrm{C}$

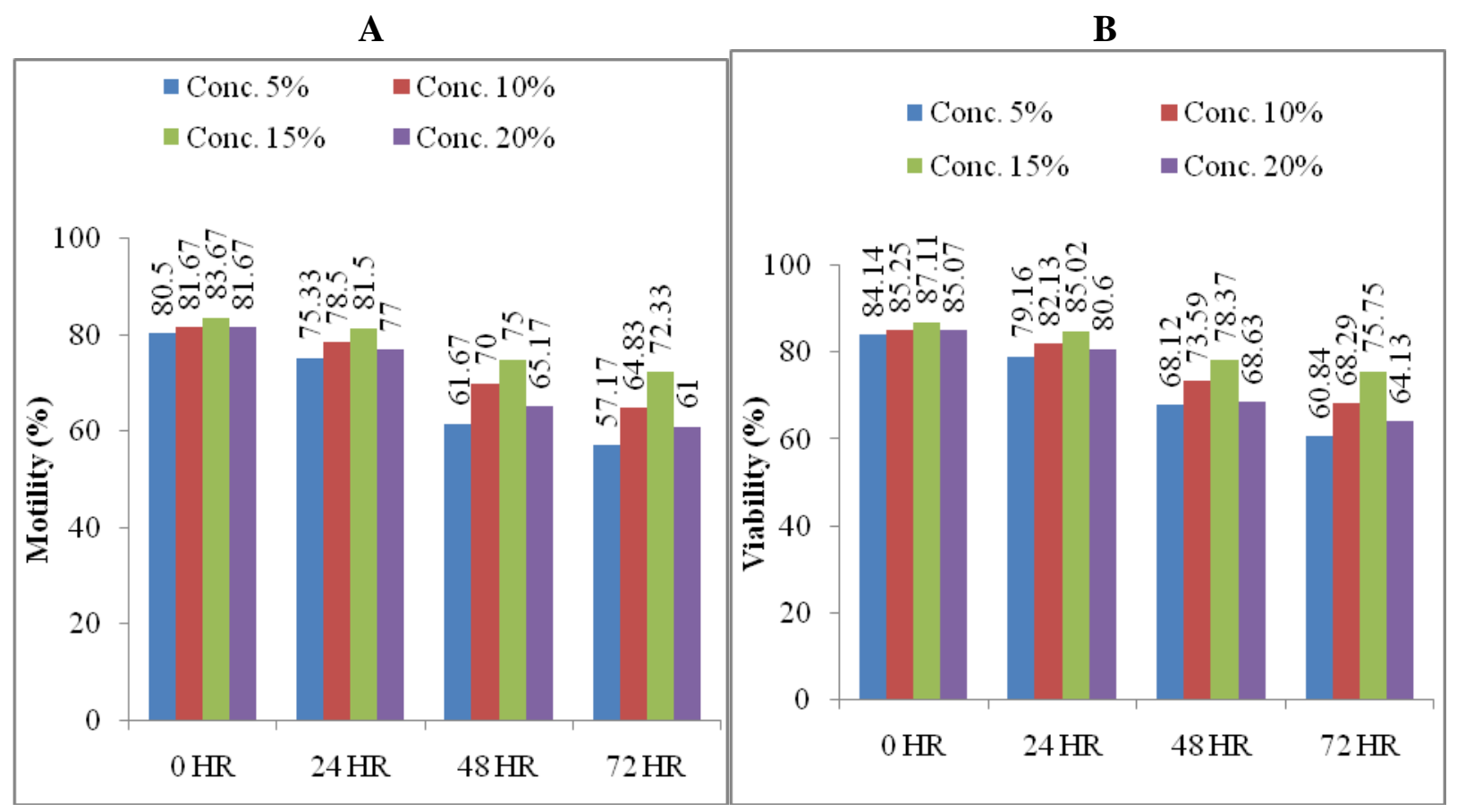

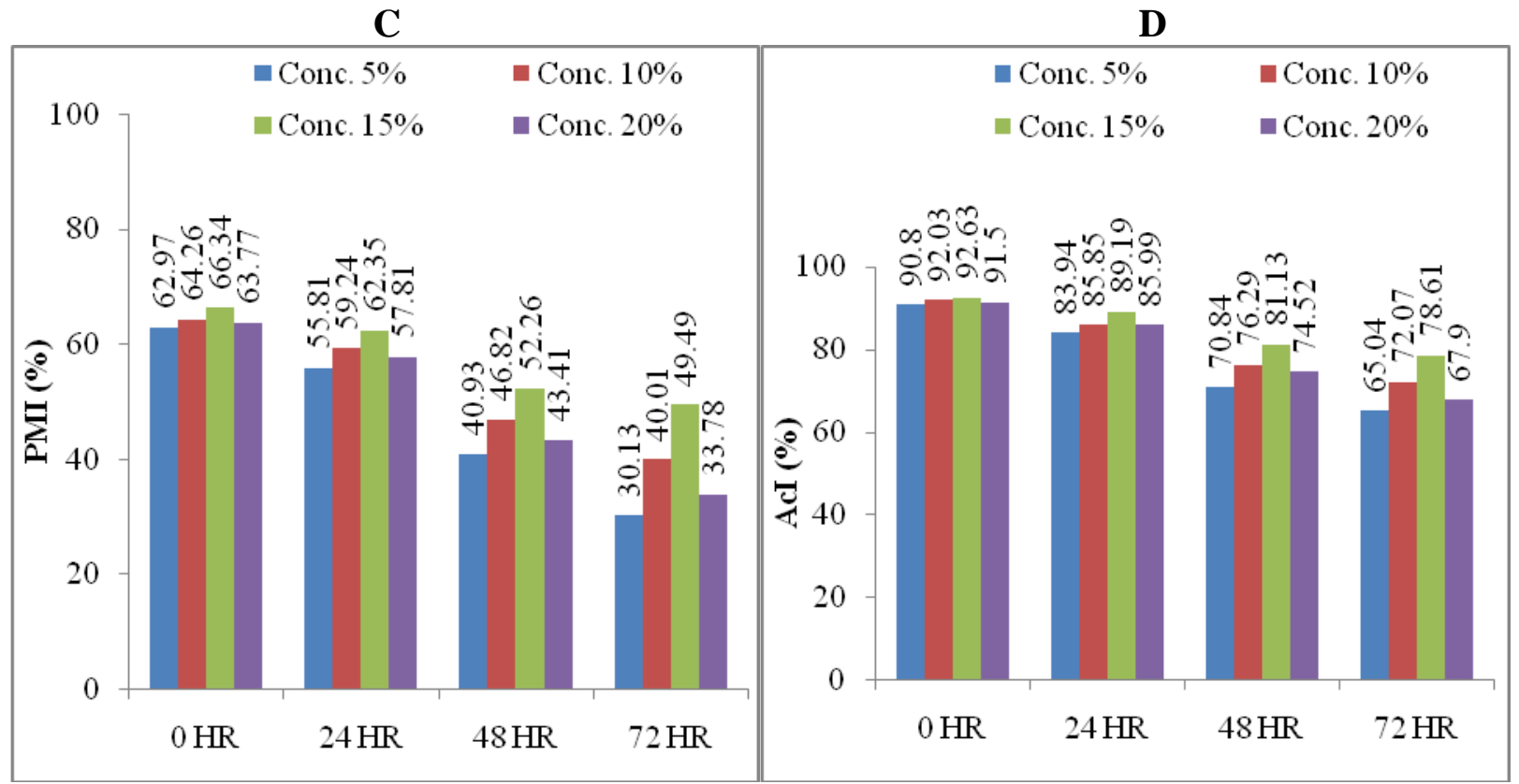


Fig. 3 Effect of supplementation of egg yolk and egg yolk plasma in Tris-citric acid fructose extender sperm motility, viability, plasma membrane integrity, Acrosomal integrity stored at $4^{\circ}$ Cat different interval of time $0 \mathrm{hr}(\mathrm{A}), 24 \mathrm{hr}(\mathrm{B}), 48 \mathrm{hr}(\mathrm{C}), 72 \mathrm{hr}(\mathrm{D})$

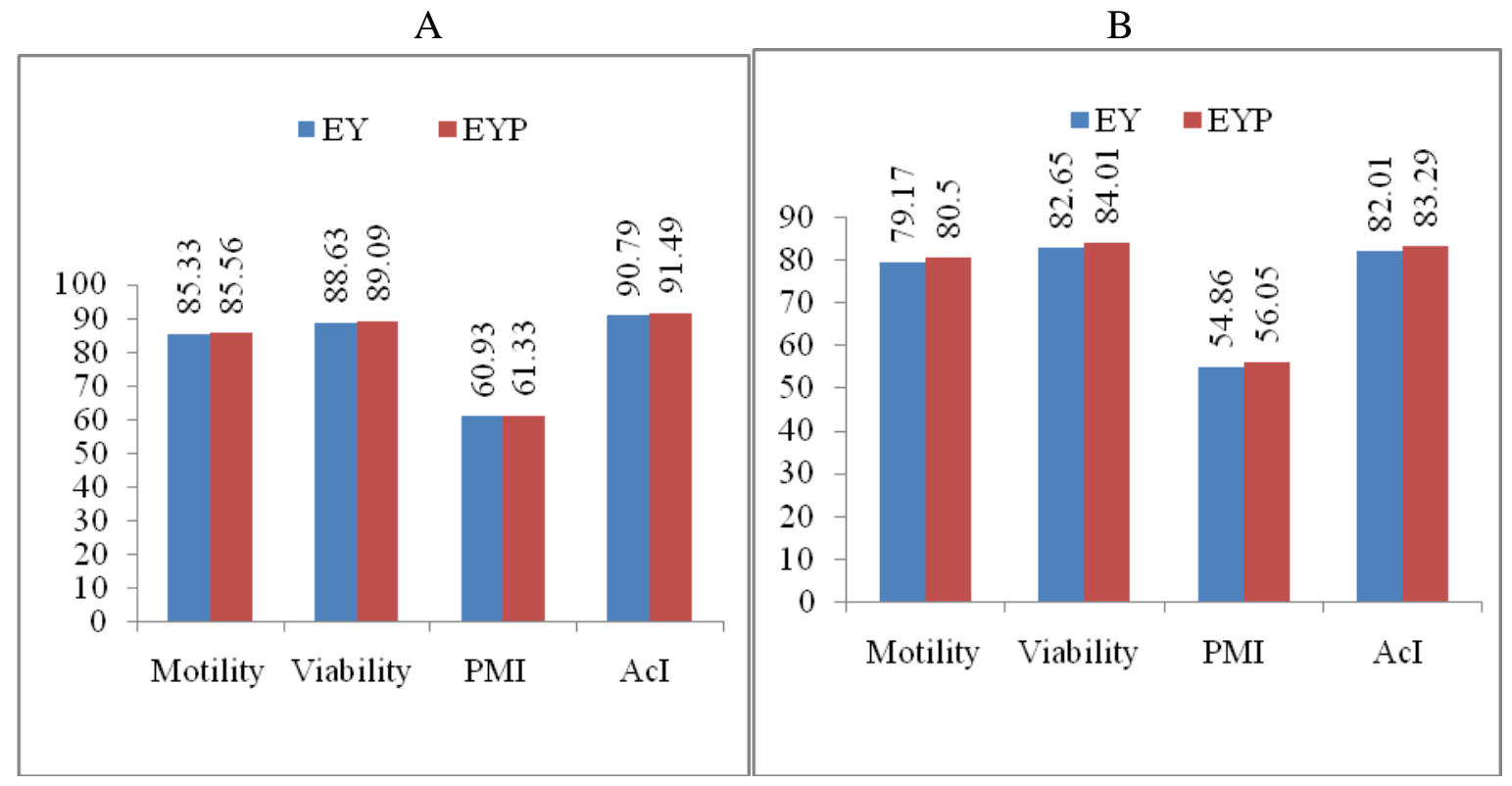

C

D

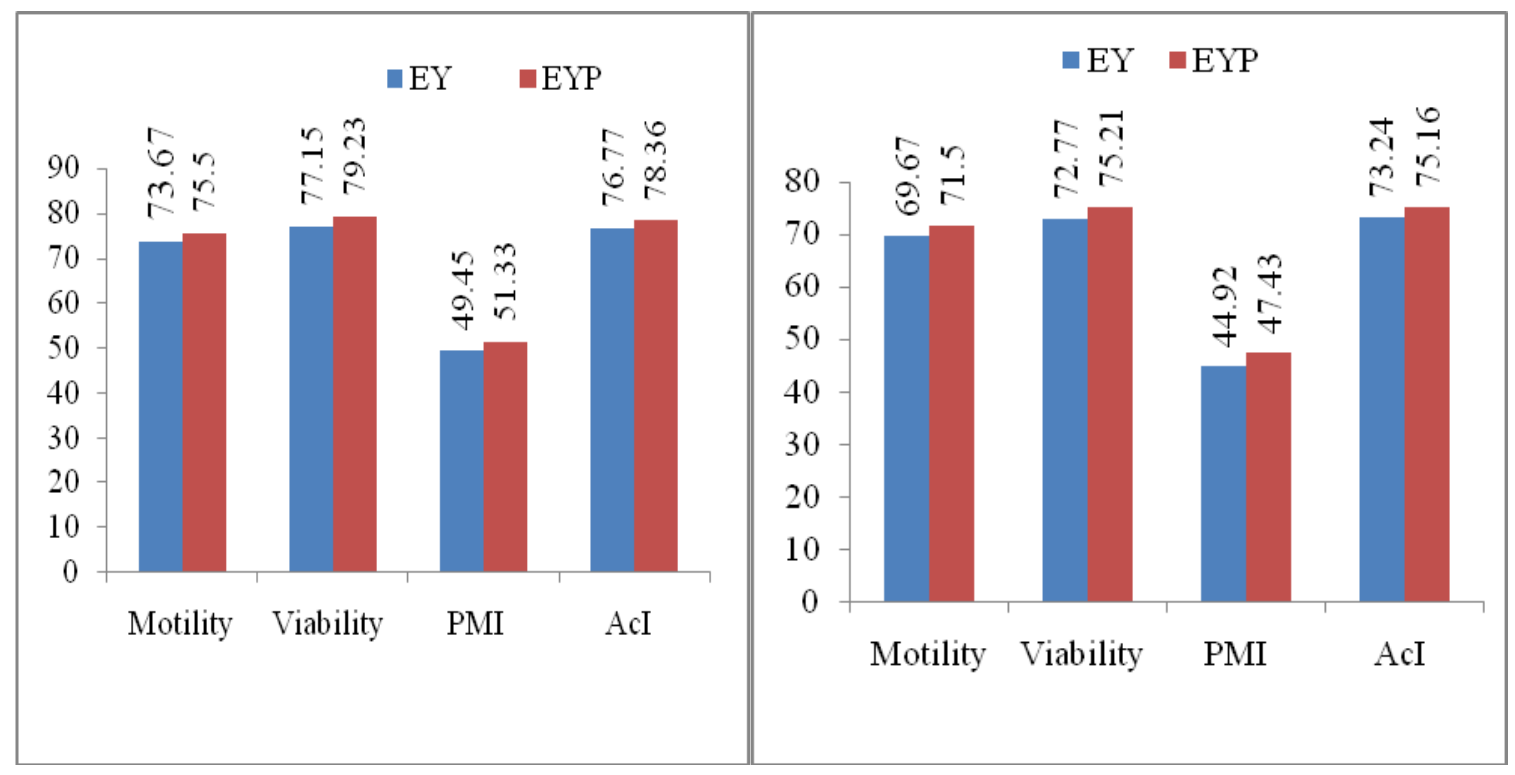


The improved viability, PMI\&AcI in TCFEYP might be due to low density lipoprotein which form complexes with seminal plasma protein, decreasing phospholipids and cholesterol efflux from the plasma membrane and elevating its resistance to cold shock (Therien et al., 1999; Bergeron et al., 2004).

As per the guidelines for frozen semen in domestic animals, the cut off varies for motility, viability, PMI \&AcI are $\geq 50 \%$, $\geq 50 \%, \geq 40 \%$ and $\geq 65 \%$, respectively. These values were almost equal to or above the recommended values in Beetal goat buck semen even after storage in $15 \%$ TCFEY \& TCFEYP at $4^{\circ} \mathrm{C}$ render it suitable to be used for AI even after $72 \mathrm{hrs}$ and higher fertility rate may be expected.

\section{Acknowledgement}

Authors are thankful to the Director Research, Guru Angad Dev Veterinary and Animal Sciences University, Ludhiana (Punjab) for facilitating this research work through financial support from RKVY project on goats.

\section{References}

Al-Faruque, M.H., Bari, F.Y., Siddiqui, M.A., and Shamsuddin, M.2007. Fertilizing capacity of buck (Capra hircus) semen frozen with different concentrations of egg yolk. Journal of the Bangladesh Agricultural University 5: 95-104.

Anand, M., Baghel, G., and Yadav, S.2017. Effect of egg yolk concentration and washing on sperm quality following cryopreservation in Barbari buck semen. Journal of Applied Animal Research45(1): 560-65.

Anonymous (2019) BAHS (Basic Animal Husbandry Statistics-2019) retrieved from http://dahd.nic.in/circulars/basic- animal-husbandary-statistics-2019

Bencharif, D., Amirat, L., Anton, M., Schmitt, E., and Desherces, S.2008. The advantages of LDL (low density lipoproteins) in the cryo preservation of canine semen, Theriogenology70:147888.

Bergeron, A., and Manjunath, P.2006. New insights towards understanding the mechanisms of sperm protection by egg yolk and milk. Molecular Reproduction and Development 73: 1338-44.

Bergeron, A., Crête, M.H., Brindle, Y., Manjunath, P. 2004. Low-density lipoprotein fraction from hen's egg yolk decreases the binding of the major proteins of bovine seminal plasma to sperm and prevents lipid efflux from the sperm membrane. Biology reproduction 70:708-17.

Bispo, C.A.S., Pugliesi, G., Galvao, P., Rodrigues, M.T., Ker, P. G., Filgueiras, B., and Carvalho, G.R. 2011. Effect of low and high egg yolk concentrations in the semen extender for goat semen cryopreservation. Small Ruminant Research 100: 54-58.

Corcini, C.D., Goularte, K.L., Bongalhardo, D.C., Lucia, Jr. T., and Jardim,R.D.2015. Effect of egg yolk plasma on dog sperm cryopreservation, Andrologia47: 1-2.

Daskin, A., and Tekin, N.1996 The effect of egg-yolk on the quality of frozen Angorabuck semen. Turk VeterinerlikveHayvancilikDergisi20(5): 395-98.

Dias, J.C.O., 2014. Adição de Ringer Lactato, citrato de sódio $2,92 \%$ e solução Trisemsêmencaprino. Dissertação (M.Sc.). Universidade Federal de Viçosa, Viçosa, MG.

Kaur, S., Cheema, R.S., Mavi, G.K., Honparkhe, M., Singh, A.K., and Gandotra, V.K. 2020. Assessment and comparison of four lab made tris-base 
extenders for preservation of Labrador retriever dog semen at $4^{\circ} \mathrm{C}$. Indian Journal of Biotechnology, 19.

Leboeuf. B., Restall, B., and Salamon, S. 2000. Production and storage of goat semen for artificial insemination. Animal Reproduction Science, 62(1-3): 113-41.

Moce, E., Blanch, E., Tomas, C., and Graham, J.K. 2010. Use of cholesterol in sperm cryopreservation: present moment and future prospects. Reproduction in Domestic Animals 45: 57-66.

Najafi, A., Zhandi, M., Towhidi, A., Sharafi, M., Sharif, A.A., Motlagh, M.K., and Martinez-Pastor, F. 2013. Trehalose and glycerol have a dose-dependent synergistic effect on the post-thawing quality of ram semen cryopreserved in a soybean lecithin-based extender. Cryobiology, 66: 275-82.

Parandekar, D.R., Pargoanker, D.R., Bakshi, S.A., and Markendya, N.M., 1991.Cytometry of buck spermatozoa. Indian Journal of Animal Reproduction
12: 95-96.

Singh, M.K., Ramachandran, N., Chauhan, M.S., and Singh, S.K. 2018. Doubling rural farmers' income through goat farming in India: prospects and potential. Indian Farming, 68(01): 7579.

Siqueira, A.P., Fonseca, J.F., Silva Filho, J.M., Bruschi, J.H., Viana, J.H.M., Palhares, M.S., Bruschi, M.C.M., and Peixoto, M.P. 2009. Parâmetrosreprodutivos de cabras Toggenburginseminadascom sêmenresfriado, apósdiluiçãoemmeio à base de gema de ovo. ArquivoBrasileiro de MedicinaVeterinária e Zootecnia, 61: 299-305.

Snedecor, G.W., and Cochran, W.G. 1994. Statistical Methods, $9^{\text {th }}$ Edn.Iowa University Press, Ames, IA, USA.

Thérien. I., Moreau, R., and Manjunath, P.1990. Bovine seminal plasma phospholipid-binding proteins stimulate phospholipid efflux from epididymal sperm. Biology of Reproduction61:59098.

\section{How to cite this article:}

Bipanpreet Singh, Daljeet Kaur, Mandeep Singla, Ranjna S. Cheema, A.P.S. Sethi, Sumit Singhal and Malik, D.S. 2020. Effects of Egg Yolk and Egg Yolk Plasma Tris-Base Extenders on Beetal Buck Semen Preservation. Int.J.Curr.Microbiol.App.Sci. 9(11): 1853-1864. doi: https://doi.org/10.20546/ijcmas.2020.911.219 